\title{
ON 3D ANTICRACK PROBLEM OF THERMOELECTROELASTICITY
}

\author{
Andrzej KACZYŃSKI \\ Faculty of Mathematics and Information Science, Warsaw University of Technology, Koszykowa 75, 00-662 Warsaw \\ akacz@mini.pw.edu.pl
}

received 8 June 2017, revised 6 June 2018, accepted 8 June 2018

\begin{abstract}
A solution is presented for the static problem of thermoelectroelasticity involving a transversely isotropic space with a heat-insulated rigid sheet-like inclusion (anticrack) located in the isotropy plane. It is assumed that far from this defect the body is in a uniform heat flow perpendicular to the inclusion plane. Besides, considered is the case where the electric potential on the anticrack faces is equal to zero. Accurate results are obtained by constructing suitable potential solutions and reducing the thermoelectromechanical problem to its thermomechanical counterpart. The governing boundary integral equation for a planar anticrack of arbitrary shape is obtained in terms of a normal stress discontinuity. As an illustration, a closed-form solution is given and discussed for a circular rigid inclusion.
\end{abstract}

Key words: Thermoelectroelasticity, Heat Flow, Anticrack, Singular Integral Equation, Thermal Stress Singularities

\section{INTRODUCTION}

Thermopiezoelectric materials are in the focus of special attention because of their potential use as functional components in many engineering applications such as sensors, actuators, smart structures, etc. (see, for instance: Rao and Sunar,1994). The main disadvantage of these materials, however, is their inherent brittleness and low fracture toughness. To promote structural mechanical reliability and lengthen service period of piezoelectric devices, thermal stress analysis is a major concern in critical design and fabrication. In particular, the presence of some original defects in structures, e.g. cracks, inclusions and voids, gives rise to high thermal stress concentration, which leads to failure of components.

Cracks (with the displacement discontinuity) and rigid lamellar inclusions called anticracks (with the traction discontinuity) represent two extreme cases of inhomogeneities that influence significantly the local fields and the overall property of the heterogeneous materials. Therefore research on these defects has great importance for structural integrity assessments.

This contribution may be treated as an extension of earlier papers (Kaczyński and Kozłowski, 2009; Kaczyński, 2014; see also extensive references therein) to the transversely isotropic thermo-piezoelectric medium. A three-dimensional problem of the determination of thermal-electric-stress state in an infinite transversely isotropic solid containing an absolutely rigid sheet-like inclusion (anticrack) in the plane of isotropy under a remote vertically uniform heat flow is considered (Fig. 1). Thermally insulated anticrack surface assumption is adopted. Moreover, the electric potential is assumed to be zero. Note that a different electric condition of electrically impermeable anticrack faces was applied in the last study by Kaczyński and Kaczyński (2017).

Problems pertinent to the present study but concerned with penny-shaped or elliptical cracks were investigated by Wang and Noda (2004), Yang et al. (2014) and Podil'chuk and Morgado (2000).
The objective of this paper is to present a general procedure for solving the posed problem and to derive the governing integral equations. Following this brief introduction, the basic equations and the potential representation of their solution are outlined in Section 2. Formulation and solution to the considered problem is given in Section 3. By using appropriate harmonic potentials, the resulting boundary-value problems involving the temperature field and induced thermal stresses are reduced to classical mixed problems of potential theory. The integro-differential and integral equations derived in the present study are, respectively, similar to the governing equations for crack and punch problems in pure elasticity. Hence, these equations can be solved by directly employing the results available in the literature. As an illustration, a complete solution expressed in elementary functions is given and discussed in Section 4 for the circularly (penny-shaped) rigid inclusion. In Section 5, the conclusions are stated.

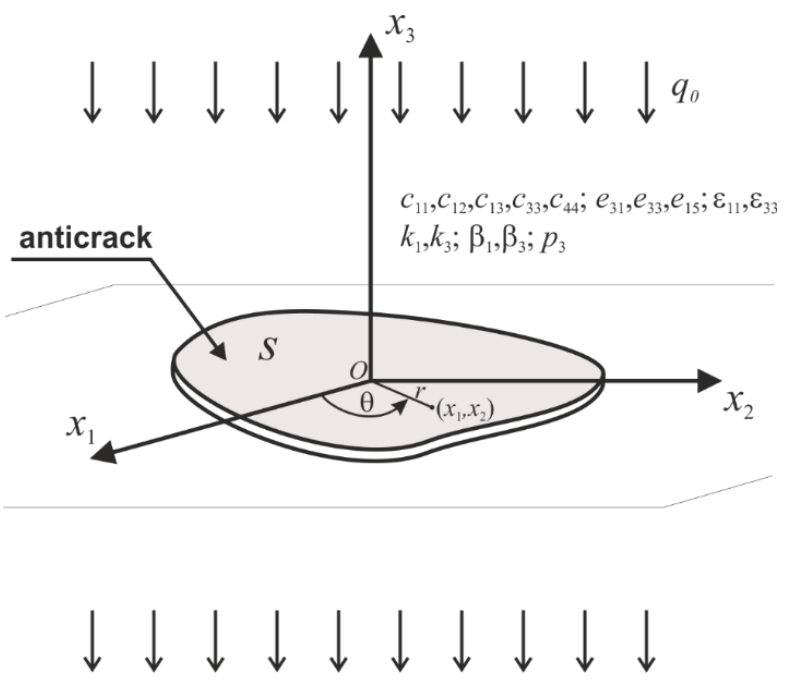

Fig. 1. An anticrack in an infinite piezoelectric medium under uniform thermal flow 


\section{BASIC EQUATIONS AND POTENTIAL SOLUTIONS}

Let us recall the fundamental equations of piezothermoelasticity for transversely isotropic bodies of the $6 \mathrm{~mm}$ class (the axis $x_{3}$ is directed along the axis of anisotropy, and the $x_{1}$ - and $x_{2}$ - axes are arbitrary oriented in the plane of isotropy) in an uncoupled static setting (Chen, 2000).

Throughout the paper, the Latin subscripts i,j are related to the Cartesian coordinates and run over 1, 2, 3, while the Greek subscripts $\alpha, \gamma$ run over 1,2 . Repeated indices imply summation and a comma denotes partial differentiation.

The constitutive relations are given by:

$$
\begin{aligned}
& \sigma_{3 \alpha}=c_{44}\left(u_{\alpha, 3}+u_{3, \alpha}\right)+e_{15} \Phi_{, \alpha}, \forall \alpha \in\{1,2\} \\
& \sigma_{33}=c_{13} u_{\gamma, \gamma}+c_{33} u_{3,3}+e_{33} \Phi_{, 3}-\beta_{3} T \\
& \sigma_{12}=c_{66}\left(u_{1,2}+u_{2,1}\right) \\
& \sigma_{11}=c_{1 \gamma} u_{\gamma, \gamma}+c_{13} u_{3,3}+e_{31} \Phi_{, 3}-\beta_{1} T \\
& \sigma_{22}=c_{13-\gamma} u_{\gamma, \gamma}+c_{13} u_{3,3}+e_{31} \Phi_{, 3}-\beta_{1} T \\
& D_{\alpha}=e_{15}\left(u_{\alpha, 3}+u_{3, \alpha}\right)-\varepsilon_{11} \Phi_{, \alpha}, \forall \alpha \in\{1,2\} \\
& D_{3}=e_{31} u_{\gamma, \gamma}+e_{33} u_{3,3}-\varepsilon_{33} \Phi_{, 3}+p_{3} T \\
& q_{\alpha}=-k_{1} T_{, \alpha}, \forall \alpha \in\{1,2\}, \quad q_{3}=-k_{3} T_{, 3}
\end{aligned}
$$

where $u_{i}, D_{i}$, and $\sigma_{i j}, q_{i}$ are components of the mechanical displacement, electric displacement, and stress, heat flux, respectively; $\Phi$ is the electric potential; $T$ is the temperature variation with $T=0$ corresponding to a traction/electric displacement-free state; $c_{11}, c_{12}, c_{13}, c_{33}, c_{44}, c_{66}=\left(c_{11}-c_{12}\right) / 2$ are the elastic constants, $e_{31}, e_{33}, e_{15}$ are the piezoelectric constants; $\varepsilon_{11}, \varepsilon_{33}$ are the dielectric permittivities; $p_{3}$ is a pyroelectric constant in the- $x_{3}$ direction; $k_{1}, k_{3}$ and $\beta_{1}, \beta_{3}$ are the thermal conductivity and stress-temperature coefficients, respectively.

The basic equations for thermopiezoelectricity in a stationary case without the body forces, electric charges and heat sources include the equilibrium equations, the electric equation and the heat conduction equation as follows:

$\sigma_{i j, j}=0, \quad D_{i, i}=0, \quad q_{i, i}=0$

Substitution of the constitutive relations (1) into (2) gives rise to a governing system of five differential equations written in the following form:

$$
\begin{aligned}
& c_{00} u_{\gamma, \gamma \alpha}+c_{66} u_{\alpha, \gamma \gamma}+c_{44} u_{\alpha, 33}+c u_{3,3 \alpha}+ \\
& e \Phi_{, 3 \alpha}=\beta_{1} T_{, \alpha}, \forall \alpha \in\{1,2\} \\
& c u_{\gamma, \gamma 3}+c_{44} u_{3, \gamma \gamma}+c_{33} u_{3,33}+e_{15} \Phi_{, \gamma \gamma}+ \\
& e_{33} \Phi_{, 33}=\beta_{3} T_{, 3} \\
& e u_{\gamma, \gamma 3}+e_{15} u_{3, \gamma \gamma}+e_{33} u_{3,33}-\varepsilon_{11} \Phi_{, \gamma \gamma}- \\
& \varepsilon_{33} \Phi_{, 33}=-p_{3} T_{, 3} \\
& T_{, \gamma \gamma}+k_{0}^{-2} T_{, 33}=0
\end{aligned}
$$

The notation for some combinations of material constants has been introduced:

$$
\begin{aligned}
& c_{00}=\left(c_{11}+c_{12}\right) / 2, \quad c=c_{13}+c_{44}, \quad e=e_{15}+e_{31}, \\
& k_{0}=\sqrt{k_{1} / k_{3}}, \quad C^{2}=c_{11} c_{33}-c_{13}\left(c_{13}+2 c_{44}\right)
\end{aligned}
$$

The general potential solutions to Eqs. (3b) and (3a) proposed by Kaczyński and Kaczyński (2017) are (see Appendix A):
$T\left(x_{1}, x_{2}, x_{3}\right)=-\frac{\partial^{2} \omega\left(x_{1}, x_{2}, z_{0}\right)}{\partial^{2} z_{0}^{2}} \mid z_{0}=k_{0} x_{3} \equiv-\omega_{, z_{0} z_{0}}$

$u_{\alpha}=\left(\psi_{1}+\psi_{2}+\psi_{3}\right)_{, \alpha}+(-1)^{\alpha} \psi_{, 3-\alpha}+c_{1} \omega_{, \alpha}$,

$\forall \alpha \in\{1,2\}$

$u_{3}=m_{i} s_{i} \psi_{i, z_{i}}-c_{2} k_{0} \omega_{, z_{0}}$

$\Phi=l_{i} s_{i} \psi_{i, z_{i}}-c_{3} k_{0} \omega_{, z_{0}}$

where $\omega, \psi_{\mathrm{i}}$ ( $\left.i \in\{1,2,3\}\right), \psi$ are five harmonic functions in the corresponding coordinate systems $\left(x_{1}, x_{2}, z_{k}\right), z_{k}=s_{k} x_{3}$, i.e., $\nabla_{z_{0}}^{2} \omega=0, \quad \nabla_{z_{i}}^{2} \psi_{i}=0, \quad \forall i \in\{1,2,3\}, \quad \nabla_{z_{4}}^{2} \psi=0$

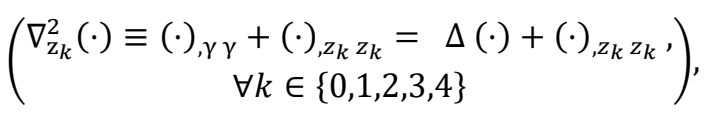

$s_{0}=k_{0}, s_{4}=\sqrt{c_{66} / c_{44}}, s_{1}, s_{2}, s_{3}$ are the roots with the positive real part of the following algebraic equation:

$a_{0} s^{6}-b_{0} s^{4}+c_{0} s^{2}-d_{0}=0$

Substituting Eqs. (5) and (6a-b) into (1), one can derive the potential expressions for the components of stress and electric displacement as follows:

$$
\begin{aligned}
& \sigma_{3 \alpha}=a_{i} s_{i} \psi_{i, z_{i} \alpha}+\delta_{1} \omega_{, z_{0} \alpha}+ \\
& +(-1)^{\alpha} s_{4} c_{44} \Psi_{, z_{4} 3-\alpha}, \quad \forall \alpha \in\{1,2\} \\
& \sigma_{33}=a_{i} \psi_{i, z_{i} z_{i}}-\delta_{3} \omega_{, z_{0} z_{0}} \\
& \sigma_{12}=c_{66}\left[2\left(\psi_{1}+\psi_{2}+\psi_{3}+c_{1} \omega\right)_{, 12}+\psi_{, 11}-\psi_{, 22}\right] \\
& \sigma_{11}=-a_{i} s_{i}^{2} \psi_{i, z_{i} z_{i}}-\delta_{0} \omega_{, z_{0} z_{0}}+ \\
& -2 c_{66}\left[\left(\psi_{1}+\psi_{2}+\psi_{3}\right)_{, 22}+\psi_{, 12}+c_{1} \omega_{, 22}\right] \\
& \sigma_{22}=-a_{i} s_{i}^{2} \psi_{i, z_{i} z_{i}}-\delta_{0} \omega_{, z_{0} z_{0}}+ \\
& -2 c_{66}\left[\left(\psi_{1}+\psi_{2}+\psi_{3}\right)_{, 11}-\psi_{, 12}+c_{1} \omega_{, 11}\right] \\
& D_{\alpha}=d_{i} s_{i} \psi_{i, z_{i} \alpha}+\tau_{1} \omega_{, z_{0} \alpha}+ \\
& +(-1)^{\alpha} s_{4} e_{15} \Psi_{, z_{4} 3-\alpha}, \forall \alpha \in\{1,2\} \\
& D_{3}=d_{i} \psi_{i, z_{i} z_{i}}-\tau_{3} \omega_{, z_{0} z_{0}}
\end{aligned}
$$

It is noted that the above general potential representation is valid only in the case of distinct material eigenvalues $s_{k} \quad(k \in$ $\{0,1,2,3,4\})$.

\section{PROBLEM FORMULATION AND SOLUTION}

Consider an infinite transversely isotropic thermopiezoelectric body that is weakened by a heat-insulated rigid inclusion (anticrack), which occupies a bounded plane area $S$ with a smooth profile in the isotropy plane $x_{3}=0$. There is a constant heat flux $\mathrm{q}(\infty)=\left[0,0,-q_{0}\right], q_{0}>0$ in the direction of the negative $x_{3}$ - symmetry axis (Fig. 1).

We deal with the boundary-value problem: find fields Tand $u_{i}$ suitable smooth on $\mathrm{R}^{3}-\mathrm{S}$ such that Eqs. (3b), (3a) and (9) hold, subject to the following global boundary conditions:

- thermal conditions at infinity (perpendicular flow of uniform heat)

$q_{1}=q_{2}=0, \quad q_{3}=-k_{3} T_{, 3}=-q_{0}$

- stress and electric-free state at infinity

$\sigma_{i j}=0, \quad D_{i}=0$ 
- mechanical conditions on $S$ (displacement free surfaces with a small vertical rigid-body translation $\varepsilon$ )

$u_{1}=u_{2}=0, u_{3}=\varepsilon$

- thermally insulated surfaces of $S$

$q_{3}=-k_{3} T_{, 3}=0$

- vanishing of electric potential on $S$

$\Phi=0$

Moreover, the parameter $\varepsilon$ will be found in solving the problem from the equilibrium condition:

$\iint_{S}\left[\sigma_{33}\left(x_{1}, x_{2}, 0^{+}\right)-\sigma_{33}\left(x_{1}, x_{2}, 0^{-}\right)\right] d x_{1} d x_{2}=0$

Making use of the superposition principle, we construct the solution of the above anticrack boundary-value problem as a sum of two components, namely:

$$
\begin{aligned}
T & =\stackrel{0}{T}+\widetilde{T}, \quad u_{i}=\stackrel{0}{u}_{i}+\tilde{u}_{i}, \quad \sigma_{i j}=\sigma_{i j}^{0}+\widetilde{\sigma}_{i j}, \\
\Phi & =\stackrel{0}{\Phi}+\widetilde{\Phi}, \quad D_{i}=\stackrel{0}{D_{i}}+\widetilde{D}_{i}
\end{aligned}
$$

where the components attached by 0 describe the basic state of defect-free solid, and the components with the tilde represent the perturbations due to the anticrack.

The results for the first 0-problem are found to be given by:

$\stackrel{0}{T}\left(x_{1}, x_{2}, x_{3}\right)=\frac{q_{0}}{k_{3}}$

$u_{\alpha}^{0}=\frac{q_{0}}{k_{3}} n_{\alpha} x_{\alpha} x_{3}, \forall \alpha \in\{1,2\}$

$\stackrel{0}{u}_{3}=\frac{q_{0}}{2 k_{3}}\left[n_{2} x_{3}^{2}-n_{1}\left(x_{1}^{2}+x_{2}^{2}\right)\right]$

$\sigma_{i j}^{0}=0, \quad \stackrel{0}{\Phi}=-\frac{q_{0}}{2 k_{3}} n_{3} x_{3}^{2}, \quad \stackrel{0}{D_{i}}=0$

where the constants $n_{i}$ are determined from the following linear system of equations:

$\left[\begin{array}{lll}2 c_{00} & c_{13} & -e_{31} \\ 2 c_{13} & c_{33} & -e_{33} \\ 2 e_{31} & e_{33} & \varepsilon_{33}\end{array}\right]\left[\begin{array}{l}n_{1} \\ n_{2} \\ n_{3}\end{array}\right]=\left[\begin{array}{l}\beta_{1} \\ \beta_{3} \\ -p_{3}\end{array}\right]$

Attention will be drawn next on the corrective solution of the perturbed problem.

The disturbing thermal field $\tilde{T}$, which is odd in $x_{3}$ and vanishes at infinity, is determined by solving Eq. (3b) in the halfspace $x_{3} \geq 0$ with the following boundary conditions:

$\tilde{T}_{, 3}=-\frac{q_{0}}{k_{3}}, \quad \forall\left(x_{1}, x_{2}, x_{3}=0^{+}\right) \in S$

$\tilde{T}=0, \quad \forall\left(x_{1}, x_{2}, x_{3}=0^{+}\right) \in R^{2}-S$

According to (5) and using the potential theory (Kellogg, 1953), the solution is written via the thermal potential $\widetilde{\omega}\left(\mathrm{x}_{1}, \mathrm{x}_{2}, \mathrm{z}_{0}\right) \quad$ as $\quad \tilde{T}\left(\mathrm{x}_{1}, \mathrm{x}_{2}, \mathrm{x}_{3}\right)=-\widetilde{\omega}_{, \mathrm{z}_{0} \mathrm{z}_{0}}, z_{0}=k_{0} x_{3}$ by assuming that:

$\widetilde{\omega}\left(x_{1}, x_{2}, z_{0}\right)=\iint_{S} \ln \left(R_{0}+z_{0}\right) \gamma\left(\xi_{1}, \xi_{2}\right) d \xi_{1} d \xi_{2}$

$\left(R_{0}=\left|\mathbf{x}_{0}-\xi\right|=\sqrt{\left(x_{1}-\xi_{1}\right)^{2}+\left(x_{2}-\xi_{2}\right)^{2}+z_{0}^{2}}\right)$

$\tilde{T}\left(x_{1}, x_{2}, x_{3}\right)=-\frac{\partial}{\partial z_{0}} \iint_{S} \frac{\gamma\left(\xi_{1}, \xi_{2}\right) d \xi_{1} d \xi_{2}}{R_{0}}$

$\tilde{T}_{, 3}=-k_{0} \frac{\partial^{2}}{\partial z_{0}^{2}} \iint_{S} \frac{\gamma\left(\xi_{1}, \xi_{2}\right) d \xi_{1} d \xi_{2}}{R_{0}}=k_{0} \Delta \iint_{S} \frac{\gamma\left(\xi_{1}, \xi_{2}\right) d \xi_{1} d \xi_{2}}{R_{0}}$

and the unknown density $\gamma$, in view of Eq. (19) 1 , satisfies the integro-differential singular equation of Newton's potential type:

$\Delta \iint_{S} \frac{\gamma\left(\xi_{1}, \xi_{2}\right) d \xi_{1} d \xi_{2}}{\sqrt{\left(x_{1}-\xi_{1}\right)^{2}+\left(x_{2}-\xi_{2}\right)^{2}}}=-\frac{q_{0}}{\sqrt{k_{1} k_{3}}}$

It is interesting to note that this equation has a similar form as that arising in Mode I crack problem. Moreover, the desired temperature has a jump on $\mathrm{S}$ :

$\tilde{T}\left(x_{1}, x_{2}, 0^{+}\right)-\tilde{T}\left(x_{1}, x_{2}, 0^{-}\right)=4 \pi \gamma\left(x_{1}, x_{2}\right)$,

$\left(x_{1}, x_{2}\right) \in S$

We proceed now to the associated problem of electroelasticity that is governed by Eqs. (3a) and (9) with the unknown quantities marked by the tilde. Because of the anti-symmetry of the temperature and stress system, and bearing in mind Eqs. (16),(17),(12),(14) and the resulting conditions for the displacements and electric potential (i.e., $\tilde{u}_{1}, \tilde{u}_{2}, \widetilde{D}_{3}$ are odd in $x_{3}$, and $\tilde{u}_{3}, \widetilde{\Phi}$ are even in $x_{3}$ ), the anticrack perturbed problem may be formulated as a mixed problem over a half-space $x_{3} \geq 0$ with the following boundary conditions:

$$
\begin{aligned}
& \tilde{u}_{\alpha}\left(x_{1}, x_{2}, x_{3}=0^{+}\right)=0, \quad \forall\left(x_{1}, x_{2}\right) \in R^{2} \quad(\alpha=1,2) \\
& \tilde{u}_{3}\left(x_{1}, x_{2}, x_{3}=0^{+}\right)=\frac{q_{0} n_{1}}{2 k_{3}}\left(x_{1}^{2}+x_{2}^{2}\right)+\varepsilon, \quad \forall\left(x_{1}, x_{2}\right) \in S \\
& \widetilde{\sigma}_{33}\left(x_{1}, x_{2}, x_{3}=0^{+}\right)=0, \quad \forall\left(x_{1}, x_{2}\right) \in R^{2}-S \\
& \widetilde{\Phi}\left(x_{1}, x_{2}, x_{3}=0^{+}\right)=0, \quad \forall\left(x_{1}, x_{2}\right) \in S \\
& \tilde{u}_{i}=0\left(|\mathrm{x}|^{-1}\right) \text { as }|\mathrm{x}|=\sqrt{x_{1}^{2}+x_{2}^{2}+x_{3}^{2}} \rightarrow \infty
\end{aligned}
$$

For the solution of this boundary-value problem we use the potential function approach based on the construction of the potentials in the general solution (6) and (9) with the knowledge of the thermal potential $\widetilde{\omega}\left(x_{1}, x_{2}, z_{0}\right)$ well suited to the boundary conditions (23). It is expedient to make the assumptions:

$\psi_{i}\left(x_{1}, x_{2}, z_{i}\right)=\tilde{b}_{i} \tilde{f}\left(x_{1}, x_{2}, z_{i}\right)+\tilde{A}_{i} \widetilde{\omega}\left(x_{1}, x_{2}, z_{i}\right)$,

$\forall i \in\{1,2,3\}$

$\psi\left(x_{1}, x_{2}, z_{4}\right) \equiv 0$

with

$\tilde{b}_{1}=l_{3} s_{3}-l_{2} s_{2}, \quad \tilde{b}_{2}=l_{1} s_{1}-l_{3} s_{3}$,

$\tilde{b}_{3}=l_{2} s_{2}-l_{1} s_{1}$

Here, $\tilde{f}\left(\mathrm{x}_{1}, \mathrm{x}_{2}, \mathrm{x}_{3}\right)$ is an unknown harmonic function and $\tilde{A}_{1}, \tilde{A}_{2}, \tilde{A}_{3}$ are constants to be determined.

Making use of equations (9) and (6b), the desired field components become:

$$
\begin{aligned}
\tilde{u}_{\alpha}= & \tilde{b}_{i}\left[\tilde{f}\left(x_{1}, x_{2}, z_{i}\right)\right]_{, \alpha}+\tilde{A}_{i}\left[\widetilde{\omega}\left(x_{1}, x_{2}, z_{i}\right)\right]_{, \alpha} \\
& +c_{1}\left[\widetilde{\omega}\left(x_{1}, x_{2}, z_{0}\right)\right]_{, \alpha}, \forall \alpha \in\{1,2\} \\
\tilde{u}_{3}= & m_{i} s_{i} \tilde{b}_{i}\left[\tilde{f}\left(x_{1}, x_{2}, z_{i}\right)\right]_{, z_{i}}+m_{i} s_{i} \tilde{A_{i}}\left[\widetilde{\omega}\left(x_{1}, x_{2}, z_{i}\right)\right]_{, z_{i}} \\
& -c_{2} k_{0}\left[\widetilde{\omega}\left(x_{1}, x_{2}, z_{0}\right)\right]_{, z_{0}} \\
\widetilde{\Phi}= & l_{i} s_{i} \tilde{b}_{i}\left[\tilde{f}\left(x_{1}, x_{2}, z_{i}\right)\right]_{, z_{i}}+l_{i} s_{i} \tilde{A}_{i}\left[\widetilde{\omega}\left(x_{1}, x_{2}, z_{i}\right)\right]_{, z_{i}} \\
& -c_{3} k_{0}\left[\widetilde{\omega}\left(x_{1}, x_{2}, z_{0}\right)\right]_{, z_{0}} \\
\widetilde{\sigma}_{3 \alpha}= & a_{i} s_{i} \tilde{b}_{i}\left[\tilde{f}\left(x_{1}, x_{2}, z_{i}\right)\right]_{, z_{i} \alpha}+a_{i} s_{i} \tilde{A}_{i}\left[\widetilde{\omega}\left(x_{1}, x_{2}, z_{i}\right)\right]_{, z_{i} \alpha} \\
& \quad+\delta_{1}\left[\widetilde{\omega}\left(x_{1}, x_{2}, z_{0}\right)\right]_{, z_{0} \alpha}, \quad \forall \alpha \in\{1,2\} \\
\widetilde{\sigma}_{33}= & a_{i} \tilde{b}_{i}\left[\tilde{f}\left(x_{1}, x_{2}, z_{i}\right)\right]_{, z_{i} z_{i}}+a_{i} \tilde{A}_{i}\left[\widetilde{\omega}\left(x_{1}, x_{2}, z_{i}\right)\right]_{, z_{i} z_{i}} \\
& \quad-\delta_{3}\left[\widetilde{\omega}\left(x_{1}, x_{2}, z_{0}\right)\right]_{, z_{0} z_{0}} \\
\widetilde{D}_{\alpha}= & d_{i} s_{i} \tilde{b}_{i}\left[\tilde{f}\left(x_{1}, x_{2}, z_{i}\right)\right]_{, z_{i} \alpha}+d_{i} s_{i} \tilde{A_{i}}\left[\widetilde{\omega}\left(x_{1}, x_{2}, z_{i}\right)\right]_{, z_{i} \alpha} \\
& +\tau_{1}\left[\widetilde{\omega}\left(x_{1}, x_{2}, z_{0}\right)\right]_{, z_{0} \alpha}, \forall \alpha \in\{1,2\} \\
\widetilde{D}_{3}= & d_{i} \tilde{b}_{i}\left[\tilde{f}\left(x_{1}, x_{2}, z_{i}\right)\right]_{, z_{i} z_{i}}+d_{i} \tilde{A}_{i}\left[\widetilde{\omega}\left(x_{1}, x_{2}, z_{i}\right)\right]_{, z_{i} z_{i}} \\
& \quad-\tau_{3}\left[\widetilde{\omega}\left(x_{1}, x_{2}, z_{0}\right)\right]_{, z_{0} z_{0}}
\end{aligned}
$$


The expressions in the above equations simplify on the plane $x_{3}=0^{+}$(where $z_{1}=z_{2}=z_{3}=z_{0}=0^{+}$) to the following:

$$
\tilde{u}_{\alpha}=\left(\tilde{A}_{1}+\tilde{A}_{2}+\tilde{A}_{3}+c_{1}\right)\left[\frac{\partial \widetilde{\omega}\left(x_{1}, x_{2}, z_{0}\right)}{\partial x_{\alpha}}\right]_{z_{0}=0},
$$$$
\forall \alpha \in\{1,2\}
$$

$$
\begin{aligned}
\tilde{u}_{3}= & m_{i} s_{i} \tilde{b}_{i}\left[\frac{\partial \tilde{f}\left(x_{1}, x_{2}, x_{3}\right)}{\partial x_{3}}\right]_{x_{3}=0^{+}} \\
& +\left(m_{i} s_{i} \tilde{A}_{i}-c_{2} k_{0}\right)\left[\frac{\partial \widetilde{\omega}\left(x_{1}, x_{2}, z_{0}\right)}{\partial z_{0}}\right]_{z_{0}=0}
\end{aligned}
$$

$$
\begin{aligned}
\widetilde{\Phi}=l_{i} s_{i} \tilde{b}_{i}\left[\frac{\partial \tilde{f}\left(x_{1}, x_{2}, x_{3}\right)}{\partial x_{3}}\right]_{x_{3}=0^{+}} \\
+\left(l_{i} s_{i} \tilde{A}_{i}-c_{3} k_{0}\right)\left[\frac{\partial \widetilde{\omega}\left(x_{1}, x_{2}, z_{0}\right)}{\partial z_{0}}\right]_{z_{0}=0}
\end{aligned}
$$

$$
\begin{aligned}
\widetilde{\sigma}_{33}= & a_{i} \tilde{b}_{i}\left[\frac{\partial^{2} \tilde{f}\left(x_{1}, x_{2}, x_{3}\right)}{\partial x_{3}^{2}}\right]_{x_{3}=0^{+}} \\
& +\left(a_{i} \tilde{A}_{i}-\delta_{3}\right)\left[\frac{\partial^{2} \widetilde{\omega}\left(x_{1}, x_{2}, z_{0}\right)}{\partial z_{0}^{2}}\right]_{z_{0}=0^{+}}
\end{aligned}
$$

$$
\begin{aligned}
\widetilde{D}_{3}=d_{i} \tilde{b}_{i} & {\left[\frac{\partial^{2} \tilde{f}\left(x_{1}, x_{2}, x_{3}\right)}{\partial x_{3}^{2}}\right]_{x_{3}=0^{+}} } \\
& +\left(d_{i} \tilde{A}_{i}-\tau_{3}\right)\left[\frac{\partial^{2} \widetilde{\omega}\left(x_{1}, x_{2}, z_{0}\right)}{\partial z_{0}^{2}}\right]_{z_{0}=0^{+}}
\end{aligned}
$$

The three unknown constants $\tilde{A}_{1}, \tilde{A}_{2}, \tilde{A}_{3}$ are to be determined from the following linear system of equations:

$$
\left[\begin{array}{lll}
1 & 1 & 1 \\
l_{1} s_{1} & l_{2} s_{2} & l_{3} s_{3} \\
a_{1} & a_{2} & a_{3}
\end{array}\right]\left[\begin{array}{l}
\tilde{A}_{1} \\
\tilde{A}_{2} \\
\tilde{A}_{3}
\end{array}\right]=\left[\begin{array}{l}
-c_{1} \\
c_{3} k_{0} \\
\delta_{3}
\end{array}\right]
$$

A glance at equations (23) and (27) reveals now that the potential $\tilde{f}$ is governed by:

- for $\left(x_{1}, x_{2}\right) \in S$

$m_{i} s_{i} \tilde{b}_{i}\left[\frac{\partial \tilde{f}\left(x_{1}, x_{2}, x_{3}\right)}{\partial x_{3}}\right]_{x_{3}=0^{+}}=r\left(x_{1}, x_{2}\right)$

- for $\left(x_{1}, x_{2}\right) \in R^{2}-S$

$\left[\frac{\partial^{2} \tilde{f}\left(x_{1}, x_{2}, x_{3}\right)}{\partial x_{3}^{2}}\right]_{x_{3}=0^{+}}=0$

where:

$r\left(x_{1}, x_{2}\right)=\tilde{\beta}\left[\frac{\partial \widetilde{\omega}\left(x_{1}, x_{2}, z_{0}\right)}{\partial z_{0}}\right]_{z_{0}=0}+\frac{q_{0} n_{1}}{2 k_{3}}\left(x_{1}^{2}+x_{2}^{2}\right)+\varepsilon$

with the following constant:

$$
\tilde{\beta}=c_{2} k_{0}-m_{i} s_{i} \tilde{A}_{i}
$$

A well-known solution to this classical boundary problem in potential theory (Kellogg, 1953) may be written as follows:

$$
\begin{aligned}
& \tilde{f}\left(x_{1}, x_{2}, x_{3}\right)= \\
& \frac{-1}{2 \pi a_{i} \tilde{b}_{i}} \iint_{S} \widetilde{\sigma}_{33}\left(\xi_{1}, \xi_{2}, 0^{+}\right) \ln \left(R_{\xi}+x_{3}\right) d \xi_{1} d \xi_{2} \\
& \left(R_{\xi}=\sqrt{\left(x_{1}-\xi_{1}\right)^{2}+\left(x_{2}-\xi_{2}\right)^{2}+x_{3}^{2}}\right)
\end{aligned}
$$

Now enforcing the displacement boundary condition (29), we arrive at the governing two-dimensional singular integral equation of Newtonian potential type to determine the normal stress $\widetilde{\sigma}_{33}^{+} \equiv \widetilde{\sigma}_{33}\left(x_{1}, x_{2}, 0^{+}\right)$on the upper side of $S$ :
$\tilde{B} \iint_{S} \frac{\widetilde{\sigma}_{33}^{+}\left(\xi_{1}, \xi_{2}\right) d \xi_{1} d \xi_{2}}{\sqrt{\left(x_{1}-\xi_{1}\right)^{2}+\left(x_{2}-\xi_{2}\right)^{2}}}=-\tilde{r}\left(x_{1}, x_{2}\right)$,

$\forall\left(x_{1}, x_{2}\right) \in S$

where the constant $\tilde{B}$ is given by:

$\tilde{B}=\frac{m_{i} s_{i} \tilde{b}_{i}}{2 \pi a_{i} \tilde{b}_{i}}$

Then, having obtained the distribution of the normal stress in the region $S$, the unknown rigid translation $\varepsilon$ can be calculated from Eq. (15) transformed to the following form:

$\iint_{S} \widetilde{\sigma}_{33}^{+}\left(x_{1}, x_{2}\right) d x_{1} d x_{2}=0$

Moreover, the main potential $\tilde{f}$ is found from Eq. (33) and the whole perturbed electroelastic fields can be obtained from relations (26).

It is worth mentioning that for a rigid inclusion with an arbitrary shape $S$, the derived governing equations (21) and (34) generally can be solved by numerical methods. However, analytical solutions to these equations are available when the anticrack is in the form of an ellipse (Rahman, 2002). For illustration, a solution will be presented for a rigid circularly shaped inclusion in the next section.

\section{EXAMPLE: CIRCULAR ANTICRACK IN A UNIFORM HEAT FLOW}

Let the anticrack is located at the circular region in the $\mathrm{x}_{1} \mathrm{Ox}_{2}$ plane, i.e.,

$$
\begin{aligned}
S=\left\{\left(x_{1}\right.\right. & \left.=r \cos \theta, x_{2}=r \sin \theta, x_{3}=0\right): \\
0 & \left.\leq r=\sqrt{x_{1}^{2}+x_{2}^{2}} \leq a \wedge 0 \leq \theta \leq 2 \pi\right\}
\end{aligned}
$$

Following along the same line of reasoning as that used in the corresponding antisymmetric anticrack problems (Kaczyński, 2014), it is possible to obtain in this case a complete solution expressed in elementary functions due to the results of advanced method in potential theory, reported by Fabrikant $(1989,1991)$. Only the final results will be presented.

Accordingly, the axially-symmetric solution to the thermal problem is given by:

$\gamma\left(x_{1}, x_{2}\right)=\tilde{\gamma}(r)=\frac{q_{0}}{\pi^{2} \sqrt{k_{1} k_{3}}} \sqrt{a^{2}-r^{2}}$,

$0 \leq r \leq a$

$\frac{\partial \widetilde{\omega}\left(r, z_{0}\right)}{\partial x_{\alpha}}=\frac{q_{0} x_{\alpha}}{\pi \sqrt{k_{1} k_{3}}}\left[-x_{3} \sin ^{-1} \frac{a}{l_{20}}+\right.$

$+\left(\sqrt{a^{2}-l_{10}^{2}}\left(1-\frac{l_{10}^{2}+2 a^{2}}{3 r^{2}}\right)+\frac{2 a^{3}}{3 r^{2}}\right], \forall \alpha \in\{1,2\}$

$\frac{\partial \widetilde{\omega}\left(r, z_{0}\right)}{\partial z_{0}}=\frac{q_{0}}{2 \pi \sqrt{k_{1} k_{3}}}\left[\left(2 a^{2}+2 z_{0}^{2}-r^{2}\right) \sin ^{-1} \frac{a}{l_{20}}\right.$

$\left.-\frac{\left(2 a^{2}-3 l_{10}^{2}\right)}{a} \sqrt{l_{20}^{2}-a^{2}}\right], \quad z_{0} \geq 0$

$\widetilde{T}\left(r, z_{0}\right)=-\frac{\partial^{2} \widetilde{\omega}}{\partial z_{0}^{2}}=$

$-\frac{2 q_{0}}{\pi \sqrt{k_{1} k_{3}}}\left(z_{0} \sin ^{-1} \frac{a}{l_{20}}-\sqrt{a^{2}-l_{10}^{2}}\right), z_{0} \geq 0$ 


\section{sciendo}

DOI 10.2478/ama-2018-0018

where Fabrikant's notation is given below:

$l_{1} \equiv l_{1}\left(a, r, x_{3}\right)=$

$\frac{1}{2}\left[\sqrt{(r+a)^{2}+x_{3}^{2}}-\sqrt{(r-a)^{2}+x_{3}^{2}}\right]$

$l_{2} \equiv l_{2}\left(a, r, x_{3}\right)=$

$\frac{1}{2}\left[\sqrt{(r+a)^{2}+x_{3}^{2}}+\sqrt{(r-a)^{2}+x_{3}^{2}}\right]$

$l_{10}=l_{1}\left(a, r, z_{0}\right), \quad l_{20}=l_{2}\left(a, r, z_{0}\right)$

along with the following properties:

$$
\begin{aligned}
& {\left[l_{1}\right]_{x_{3}=0}=\left[l_{10}\right]_{z_{0}=0}=\min (a, r)} \\
& {\left[l_{2}\right]_{x_{3}=0}=\left[l_{20}\right]_{z_{0}=0}=\max (a, r)}
\end{aligned}
$$
is:

In turn, the analytical solution to the governing equation (34)

$$
\widetilde{\sigma}_{33}^{+}(r)=\frac{\beta_{3}^{(e p)} q_{0}}{\pi} \frac{2 a^{2}-3 r^{2}}{\sqrt{a^{2}-r^{2}}}, \quad 0 \leq r<a
$$

where (see Eqs.(18) and (32)):

$\beta_{3}^{(e p)}=\frac{2 a_{i} \tilde{b}_{i}}{3 m_{j} s_{j} \tilde{b}_{j}}\left(\frac{2 n_{1}}{k_{3}}-\frac{\widetilde{\beta}}{\sqrt{k_{1} k_{3}}}\right)$

and the vertical rigid displacement is found as:

$\varepsilon=-\frac{a^{2} q_{0}}{3}\left(\frac{\widetilde{\beta}}{\sqrt{k_{1} k_{3}}}+\frac{n_{1}}{k_{3}}\right)$

The primary harmonic potential for the electroelastic perturbed problem is obtained by calculating integral (32) with the use of Eq. (40). As a result, we find that for $x_{3} \geq 0$ :

$$
\begin{aligned}
& \tilde{f}\left(x_{1}, x_{2}, x_{3}\right)= \\
& -\frac{\beta_{3}^{(e p)} q_{0}}{2 \pi^{2} a_{i} \widetilde{b}_{i}}\left[x_{3} \sin ^{-1} \frac{a}{l_{2}}\left(a^{2}-\frac{3}{2} r^{2}+x_{3}^{2}\right)+\right. \\
& \left.+\sqrt{a^{2}-l_{1}^{2}}\left(5 r^{2}+\frac{1}{3} a^{2}-l_{2}^{2}-\frac{11}{6} l_{1}^{2}\right)\right]
\end{aligned}
$$

and

$\frac{\partial \tilde{f}\left(x_{1}, x_{2}, x_{3}\right)}{\partial x_{3}}=$

$-\frac{\beta_{3}^{(e p)} q_{0}}{2 \pi a_{i} \widetilde{b}_{i}}\left[\left(a^{2}-\frac{3}{2} r^{2}+3 x_{3}^{2}\right) \sin ^{-1} \frac{a}{l_{2}}+\right.$

$\left.-\frac{3\left(2 a^{2}-3 l_{1}^{2}\right) \sqrt{l_{2}^{2}-a^{2}}}{2 a}\right]$

$\frac{\partial^{2} \tilde{f}\left(x_{1}, x_{2}, x_{3}\right)}{\partial x_{3}^{2}}=$

$-\frac{\beta_{3}^{(e p)} q_{0}}{\pi a_{i} \widetilde{b}_{i}}\left[3 x_{3} \sin ^{-1} \frac{a}{l_{2}}-3 \sqrt{a^{2}-l_{1}^{2}}+\frac{a^{2} \sqrt{a^{2}-l_{1}^{2}}}{l_{2}^{2}-l_{1}^{2}}\right]$

Having the exact expressions for the governing harmonic functions and their derivatives as shown in Eqs. (45) and (38), the full -space piezothermoelastic field can be obtained simply from formulas (26). The derivation is omitted here to save the space of the paper. To investigate the singular behaviour of the thermalelectric-stress field near the disc edge, however, the solution in the inclusion plane $x_{3}=0^{ \pm}$is given below:

$T\left(r, 0^{ \pm}\right)=\left\{\begin{array}{cl} \pm \frac{2 q_{0}}{\pi \sqrt{k_{1} k_{3}}} \sqrt{a^{2}-r^{2}} & 0 \leq r \leq a \\ 0 & r>a\end{array}\right.$

$$
\begin{aligned}
& q_{r}\left(r, 0^{ \pm}\right)=-k_{1} \frac{\partial T\left(r, 0^{ \pm}\right)}{\partial r}= \\
& =\left\{\begin{array}{lc} 
\pm \frac{2 q_{0}}{\pi} \sqrt{\frac{k_{1}}{k_{3}} \frac{r}{\sqrt{a^{2}-r^{2}}}} & 0 \leq r \leq a \\
0 & r>a
\end{array}\right. \\
& q_{3}\left(r, 0^{ \pm}\right)=-k_{3} T_{, 3}\left(r, 0^{ \pm}\right)= \\
& \quad=\left\{\begin{array}{lcc}
0 & 0 \leq r<a \\
\frac{2 q_{0}}{\pi}\left(\sin ^{-1} \frac{a}{r}-\frac{a}{\sqrt{r^{2}-a^{2}}}\right)-q_{0} & r>a
\end{array}\right.
\end{aligned}
$$$$
u_{1}\left(r, 0^{ \pm}\right)=u_{2}\left(r, 0^{ \pm}\right)=0 \quad 0 \leq r<\infty
$$$$
u_{3}\left(r, 0^{ \pm}\right)=\left\{\begin{array}{l}
\frac{\varepsilon}{\pi}\left(\varepsilon+\frac{q_{0} n_{1}}{2 k_{3}} r^{2}\right) \sin ^{-1} \frac{a}{r}+ \\
-\frac{q_{0} n_{1}}{\pi k_{3}} \sqrt{r^{2}-a^{2}}-\frac{q_{0} n_{1}}{2 k_{3}} r^{2} \quad r>a
\end{array}\right.
$$$$
\sigma_{33}\left(r, 0^{ \pm}\right)= \begin{cases} \pm \frac{\beta_{3}^{(e p)} q_{0}}{\pi} \frac{2 a^{2}-3 r^{2}}{\sqrt{a^{2}-r^{2}}} & 0 \leq r<a \\ 0 & r>a\end{cases}
$$

$\sigma_{3 r}\left(r, 0^{ \pm}\right)=\sigma_{31}\left(r, 0^{ \pm}\right) \cos \theta+\sigma_{32}\left(r, 0^{ \pm}\right) \sin \theta=$

$=\left\{\begin{array}{l}\beta^{(e p)} q_{0} r \\ \frac{2 q_{0}}{\pi}\left(\beta^{(e p)} r \sin ^{-1} \frac{a}{r}-\frac{\beta_{r}^{(e p)} a^{3}}{r \sqrt{r^{2}-a^{2}}}-\frac{\beta^{(e p)} a \sqrt{r^{2}-a^{2}}}{r}\right) \\ r>a\end{array}\right.$

$$
\Phi\left(r, 0^{ \pm}\right)=0 \quad 0 \leq r<\infty
$$

$D_{r}\left(r, 0^{ \pm}\right)=D_{1}\left(r, 0^{ \pm}\right) \cos \theta+D_{2}\left(r, 0^{ \pm}\right) \sin \theta=$

$=\left\{\begin{array}{l}\tilde{\beta}^{(e p)} q_{0} r \\ \frac{2 q_{0}}{\pi}\left(\tilde{\beta}^{(e p)} r \sin ^{-1} \frac{a}{r}-\frac{\tilde{\beta}_{r}^{(e p)} a^{3}}{r \sqrt{r^{2}-a^{2}}}-\frac{\tilde{\beta}^{(e p)} a \sqrt{r^{2}-a^{2}}}{r}\right)\end{array}\right.$ $r>a$

$D_{3}\left(r, 0^{ \pm}\right)=$

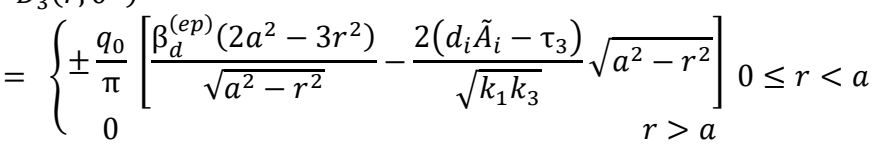
where:

$\beta^{(e p)}=\frac{3 \beta_{3}^{(e p)} a_{j} s_{j} \tilde{b}_{j}}{4 a_{i} \tilde{b}_{i}}-\frac{\left(a_{k} s_{k} \tilde{A}_{k}+\delta_{1}\right)}{2 \sqrt{k_{1} k_{3}}}$

$\beta_{r}^{(e p)}=\frac{a_{j} s_{j} \tilde{b}_{j}}{3 m_{i} s_{i} \tilde{b}_{i}}\left(\frac{2 n_{1}}{k_{3}}-\frac{\tilde{\beta}}{\sqrt{k_{1} k_{3}}}\right)$

$\tilde{\beta}^{(e p)}=\frac{3 \beta_{3}^{(e p)} d_{j} s_{j} \tilde{b}_{j}}{4 d_{i} \tilde{b}_{i}}-\frac{\left(d_{k} s_{k} \tilde{A}_{k}+\tau_{1}\right)}{2 \sqrt{k_{1} k_{3}}}$

$\tilde{\beta}_{r}^{(e p)}=\frac{d_{j} s_{j} \tilde{b}_{j}}{3 m_{i} s_{i} \tilde{b}_{i}}\left(\frac{2 n_{1}}{k_{3}}-\frac{\tilde{\beta}}{\sqrt{k_{1} k_{3}}}\right)$

$\beta_{d}^{(e p)}=\frac{d_{j} \tilde{b}_{j}}{a_{i} \tilde{b}_{i}} \beta_{3}^{(e p)}$

Analyzing the above expressions, we reveal that

1. The anticrack $S$ obstructs locally the heat flow, producing the jump of temperature and the drastic change of its gradient on the surface near the anticrack front.

2. The normal stress $\sigma_{33}$ and electric displacement $D_{3}$ suffer jumps across $S$ and exhibit the inverse square-root singularity at $r=a^{-}$. This indicates a mechanism of failure in the form of material separation from the surface of the rigid inclusion 
According to Kaczyński and Kaczyński (2017), material described by the stress and electric displacement singularity coefficients given by:

$S_{\mathrm{I}}^{ \pm}=\lim _{r \rightarrow a^{-}} \sqrt{2 \pi(a-r)} \sigma_{33}\left(r, 0^{ \pm}\right)=$

$\mp \frac{\beta_{3}^{(e p)} q_{0} a \sqrt{a}}{\sqrt{\pi}}$

$S_{\mathrm{I}}^{ \pm(e d)}=\lim _{r \rightarrow a^{-}} \sqrt{2 \pi(a-r)} D_{3}\left(r, 0^{ \pm}\right)=$

$\mp \frac{\beta_{d}^{(e p)} q_{0} a \sqrt{a}}{\sqrt{\pi}}$

3. Another mechanism controlling the material cracking around the anticrack front is Mode II (edge-sliding) described by the thermal and electric stress intensity factors:

$K_{\mathrm{II}}^{(e)}=\lim _{r \rightarrow a^{+}} \sqrt{2 \pi(r-a)} \sigma_{3 r}(r, 0)$

$=-\frac{2 \beta_{r}^{(e p)} q_{0} a \sqrt{a}}{\sqrt{\pi}}$

$K_{\mathrm{II}}^{(e d)}=\lim _{r \rightarrow a^{+}} \sqrt{2 \pi(r-a)} D_{r}(r, 0)$

$=-\frac{2 \widetilde{\beta}_{r}^{(e p)} q_{0} a \sqrt{a}}{\sqrt{\pi}}$

These parameters can be used in conjunction with a suitable force criterion of fracture.

\section{CONCLUSIONS}

The three-dimensional thermal stress problem for an insulated rigid inclusion obstructing a uniform heat flux in an infinite linear transversely isotropic thermopiezoelectric medium has been investigated. The case where the electric potential on the anticrack surface is equal to zero was considered. Using the potential function method, the problem involving the inclusion of arbitrary shape has been reduced to classical boundary problems of potential theory. Specifically, with the knowledge of the steady-state temperature distribution, the governing equation was derived, which is similar to that reported in the literature on contact problems in elasticity. In particular, for a circularly shaped inclusion, the solution was obtained in terms of elementary functions. Exact expressions for the thermo-electro-elastic field at the plane of anticrack surface were derived and interpreted from the point of view of linear fracture mechanics. The results obtained are new to the literature and can serve as a benchmark to various numerical analysis.

\section{Appendix A}

The material coefficients in characteristic equation (8) are as follows (see (4)):

$$
\begin{aligned}
a_{0}= & c_{44}\left(c_{33} \varepsilon_{33}+e_{33}^{2}\right) \\
& +e_{33}\left(2 c_{44} e_{15}+c_{11} e_{33}-2 c e\right) \\
b_{0}= & c_{33}\left(c_{44} \varepsilon_{11}+e^{2}\right)+\varepsilon_{33} C^{2} \\
& +e_{33}\left(2 c_{44} e_{15}+c_{11} e_{33}-2 c e\right) \\
c_{0}= & c_{44}\left(c_{11} \varepsilon_{33}+e^{2}\right)+\varepsilon_{11} C^{2} \\
& +e_{15}\left(2 c_{11} e_{33}+c_{44} e_{15}-2 c e\right) \\
d_{0}= & c_{11}\left(c_{44} \varepsilon_{11}+e_{15}^{2}\right)
\end{aligned}
$$

constants appearing in representations (6) and (9) are listed below:

$$
\begin{aligned}
& m_{i}=\frac{-c_{44} e_{33} s_{i}^{4}+\left(c_{11} e_{33}+c_{44} e_{15}-c e\right) s_{i}^{2}-c_{11} e_{15}}{s_{i}^{2}\left[\left(c e_{33}-e c_{33}\right) s_{i}^{2}-\left(c e_{15}-e c_{44}\right)\right]} \\
& l_{i}=\frac{c_{44} c_{33} s_{i}^{4}-\left(c^{2}-2 c_{44}^{2}\right) s_{i}^{2}+c_{11} c_{44}}{s_{i}^{2}\left[\left(c e_{33}-e c_{33}\right) s_{i}^{2}-\left(c e_{15}-e c_{44}\right)\right]} \\
& a_{i}=c_{44}\left(1+m_{i}\right)+e_{15} l_{i} \\
& d_{i}=e_{15}\left(1+m_{i}\right)-\varepsilon_{11} l_{i} \\
& \delta_{0}=c_{11} c_{1}+k_{0}^{2}\left(c_{13} c_{2}+e_{31} c_{3}\right)-\beta_{1} \\
& \delta_{1}=k_{0}\left[c_{44}\left(c_{1}-c_{2}\right)-e_{15} c_{3}\right] \\
& \delta_{3}=c_{13} c_{1}+k_{0}^{2}\left(c_{33} c_{2}+e_{33} c_{3}\right)-\beta_{3} \\
& \tau_{1}=k_{0}\left[e_{15}\left(c_{1}-c_{2}\right)+\varepsilon_{11} c_{3}\right] \\
& \tau_{3}=e_{31} c_{1}+k_{0}^{2}\left(e_{33} c_{2}-\varepsilon_{33} c_{3}\right)+p_{3}
\end{aligned}
$$

Moreover, the constants $c_{i}$ are given from the solution of the following linear system:

$$
\begin{aligned}
& {\left[\begin{array}{lll}
c_{11}-c_{44} k_{0}^{2} & c k_{0}^{2} & e k_{0}^{2} \\
c & c_{33} k_{0}^{2}-c_{44} & e_{33} k_{0}^{2}-e_{15} \\
e & e_{33} k_{0}^{2}-e_{15} & \varepsilon_{11}-\varepsilon_{33} k_{0}^{2}
\end{array}\right]\left[\begin{array}{l}
c_{1} \\
c_{2} \\
c_{3}
\end{array}\right]} \\
& =\left[\begin{array}{l}
\beta_{1} \\
\beta_{3} \\
-p_{3}
\end{array}\right]
\end{aligned}
$$

\section{REFERENCES}

1. Chen W.Q., (2000), On the general solution for piezothermoelasticity for transverse isotropy with application, Journal of Applied Mechanics, 67, 705-711.

2. Fabrikant V.I. (1989), Applications of Potential Theory in Mechanics: A Selection of New Results, Kluwer Academic Publishers, Dordrecht.

3. Fabrikant V.I. (1991), Mixed Boundary Value Problems of Potential Theory and Their Applications in Engineering, Kluwer Academic Publishers, Dordrecht.

4. Kaczyński A. (2014), Thermal stress analysis of a three-dimensional anticrack in a transversely isotropic solid, International Journal of Solids and Structures, 51, 2382-2389.

5. Kaczyński A., Kaczyński B. (2017), On 3D problem of an anticrack under vertically uniform heat flow in a transversely isotropic electrothermo-elastic space, European Journal of Mechanics A/Solids, 66,15-25.

6. Kaczyński A., Kozłowski W. (2009), Thermal stresses in an elastic space with a perfectly rigid flat inclusion under perpendicular heat flow, International Journal of Solids and Structures, 46, 1772-1777.

7. Kellogg O.D. (1953), Foundation of Potential Theory, Dover, New York.

8. Podil'chuk Yu.,N., Morgado A.H.P. (2000), Stress distribution in a transversally isotropic piezoceramic body with an elliptic crack in a uniform heat flow, International Applied Mechanics, 36(2), 203-215.

9. Rahman M. (2002), A rigid elliptical disc-inclusion, in an elastic solid, subjected to a polynomial normal shift, Journal of Elasticity, 66, 207-235.

10. Rao S.S., Sunar M. (1994), Piezoelectricity and its use in disturbance sensing and control of flexible structures: a survey, Applied Mechanics Reviews, 47, 113-123.

11. Wang B.,L., Noda N. (2004), Exact thermoelectroelasticity solution for a penny-shaped in piezoelectric materials, Journal of Thermal Stresses, 27, 241-251.

12. Yang J., Jin X., Jin N. (2014), A penny-shaped crack in an infinite linear transversely isotropic medium subjected to uniform antisymmetric heat flux: Closed form-solution, European Journal of Mechanics A/Solids, 47, 254-270. 